\title{
Klotho-deficient mice are resistant to bone loss induced by unloading due to sciatic neurectomy
}

\author{
T Yamashita ${ }^{1}$, I Sekiya ${ }^{1}$, N Kawaguchi ${ }^{1,2}$, K Kashimada ${ }^{1}$, \\ A Nifuji ${ }^{1}$, Y-I Nabeshima ${ }^{2,3}$ and $\mathbf{M ~ N o d a}^{1}$ \\ ${ }^{1}$ Department of Molecular Pharmacology, Medical Research Institute, Tokyo Medical and Dental University, Tokyo 101-0062, Japan \\ ${ }^{2}$ Core Research for Evolutional Science and Technology, Japan Science and Technology Corp., Saitama 332-0012, Japan \\ ${ }^{3}$ Department of Pathology and Tumor Biology, Graduate School of Medicine, Kyoto University, Kyoto 606-8501, Japan \\ (Requests for offprints should be addressed to M Noda, Department of Molecular Pharmacology, Medical Research Institute, Tokyo Medical and Dental \\ University, 2-3-10 Kanda-Surugadai, Chiyoda-ku, Tokyo 101-0062, Japan; Email: noda.mph@mri.tmd.ac.jp)
}

\begin{abstract}
Unloading induces bone loss as seen in experimental animals as well as in space flight or in bed-ridden conditions; however, the mechanisms involved in this phenomenon are not fully understood. Klotho mutant mice exhibit osteopetrosis in the metaphyseal regions indicating that the klotho gene product is involved in the regulation of bone metabolism. To examine whether the klotho gene product is involved in the unloading-induced bone loss, the response of the osteopetrotic cancellous bones in these mice was investigated. Sciatic nerve resection was conducted using klotho mutant $(\mathrm{kl} / \mathrm{kl})$ and control heterozygous mice $(+/ k l)$ and its effect on bone was examined by micro-computed tomography $(\mu \mathrm{CT})$. As reported previously for wild-type mice $(+/+)$, about $30 \%$ bone loss was
\end{abstract}

induced in heterozygous mice $(+/ k l)$ by unloading due to neurectomy within 30 days of the surgery. By contrast, $\mathrm{kl} / \mathrm{kl}$ mice were resistant against bone loss induced by unloading after neurectomy. Unloading due to neurectomy also induced a small but significant bone loss in the cortical bone of the mid-shaft of the femur in the heterozygous mice; no reduction in the cortical bone was observed in $\mathrm{kl} / \mathrm{kl}$ mice. These results indicate that klotho mutant mice are resistant against bone loss induced by unloading due to neurectomy in both cortical and trabecular bone and indicate that klotho is one of the molecules involved in the loss of bone by unloading.

Journal of Endocrinology (2001) 168, 347-351

\section{Introduction}

Unloading-induced enhancement of bone loss has been seen in many cases, including space flight, in long-term bed-ridden patients (Morey \& Baylink 1978), and in tail-suspension in rodents (Wronski \& Morey 1982, Simske et al. 1992). Unloading by hindlimb immobilization due to neurectomy also induces bone loss (Turner \& Bell 1986, Wakley et al. 1988). This reduction in bone mass is caused by an initial decrease in bone formation and subsequent increase in the osteoclastic bone resorption (Weinreb et al. 1989, Murakami et al. 1994, Zeng et al. 1996, Sakai et al. 1996, Shen et al. 1997). Neurectomy decreases the parameters of endosteal bone formation in the tibiae of mice with a subsequent increase or no increase in the eroded surface or the size of medullary cavity at 4 weeks after surgery (Kodama et al. 1999). However, the molecules involved in unloading-induced bone loss have not fully been investigated. Klotho mutant mice ( $k l / k l$ mice) exhibit osteopetrosis in the metaphyseal region of femur, tibia and in both the cephalic and caudal ends of vertebral bodies (Kuro-o et al. 1997, Yamashita et al. 1998, Kawaguchi et al. 1999). Osteoblasts and osteoclasts do not by themselves express the klotho gene product (Kuro-o et al. 1997); however, the deficiency of this gene appears to influence the activity of at least one type of bone cells, namely the osteoclasts. Marrow ablation studies indicated that resorption of the marrow bone was impaired in klotho mutant mice (Yamashita et al. 2000b), whereas formation of new bone marrow after ablation was not impaired (Yamashita et al. 2000b). This interesting in vivo feature on the defect in osteoclastic bone resorption leads to the characteristic structures of cancellous bones in the klotho mutant mice (Yamashita et al. 2000a). As these mice are resistant to the resorption of bones, klotho could be involved in regulation of bone metabolism by favoring bone resorption. In this paper, we investigated whether unloading-induced bone loss in the hind limb of neurectomized mice is influenced by the lack of the klotho gene product. 


\section{Materials and Methods}

\section{Animals}

Klotho mutant $(k l / k l)$ mice and heterozygous $(+/ k l)$ littermates were created by mating heterozygous mice having mutations in the klotho gene locus $(\mathrm{C} 3 \mathrm{H} / \mathrm{J}$ and $\mathrm{C} 57 \mathrm{BL} / 6 \mathrm{~J}$ mixed background). All experiments were performed according to the Institute's guidance for the care and use of laboratory animals.

\section{Neurectomy}

A total of $10 \mathrm{kl} / \mathrm{kl}$ and $16+/ \mathrm{kl}$ heterozygous mice were used for this study (4-6-weeks-old at the start of experiments). Animals were anesthetized with sodium pentobarbital $(40 \mu \mathrm{g} / \mathrm{g}$ body weight). A $5-6 \mathrm{~mm}$ segment of right sciatic nerve was resected. As a control, the left sciatic nerve was exposed surgically but was not resected. At 13 and 30 days after surgery, mice were killed and the femora and tibiae were dissected and subjected to morphometry.

\section{Morphometric analysis}

For micro-computed tomography $(\mu \mathrm{CT})$ analysis, we used Musashi (NS-ELEX, Tokyo, Japan) to analyze bone in a mid-sagittal plane at the distal end of the femur. The sampling sites were determined on the basis of the scout view of every sample taken before the CT measurement to assure the consistency and reproducibility of the analyses. In addition, tomographs of a cross-sectional plane of the mid-shaft of the femur were obtained by using $\mu \mathrm{CT} 20$ (SCANCO Medical, Basserdorf, Switzerland). Thresholds were set at 140 and 165 for Musashi and $\mu$ CT20, respectively (Yamashita et al. 2000a). Two-dimensional CT images were binarized, and bone areas were quantified by using a Luzex-F image-analyser (NIRECO, Tokyo, Japan). Trabecular bone volume was analyzed in a rectangle tissue area $(0.28 \times 2.8 \mathrm{~mm})$ in the metaphyseal region $0.1 \mathrm{~mm}$ away from the growth plate at the distal end of the femora. For morphometry of cortical bones, we used tomograms of the cross-section of the mid-shaft of the femur. Cortical bone area (B.Ar) and bone marrow area (Ma.Ar) were measured.

\section{Statistical analysis}

All data are expressed as means \pm s.E.M. The difference in the categorical values were compared by Wilcoxon's signed rank test, whereas continuous data were compared by ANOVA and Bonferroni correction for multiple comparisons. $P$ values $<0.05$ were considered significant.

\section{Results}

As a control, we used $+/ k l$ mice in our experiments. In these mice trabecular bones in the femora on the neurec- tomized side became slightly sparse on day 13 , and by day 30 the trabecular bone in the metaphyses was much sparser and thinner in the neurectomized side compared with the contralateral sham-operated side (Fig. 1). In contrast to $+/ k l$ heterozygotic mice, the trabecular bones in the metaphyseal regions of $\mathrm{kl} / \mathrm{kl}$ mice were relatively dense as reported previously (Yamashita et al. 2000a, 2000b) (Fig. 1). In these mice the neurectomy did not appreciably alter the trabecular pattern compared with the contralateral side on day 13 and 30 after the surgery (Fig. 1). Similar to the observation in the femora, neurectomy also induced sparsity in the trabecular bone of the tibiae in $+/ k l$ type but not in $k l / k l$ mice based on $\mu \mathrm{CT}$ analysis. There was no significant difference in the femoral length between $+/ k l$ and $k l / k l$ mice at 30 days after surgery (data not shown).

Quantification of the trabecular bone volume (BV/TV) was conducted and the ratios (right/left) of bone volume $(\mathrm{BV} / \mathrm{TV})$ in neurectomized side (right) femur over that (BV/TV) in sham-operated (left) femur were calculated. As shown in Fig. 2, bone volume in the $+/ k l$ mice was reduced by approximately $13 \%$ by day 13 and by $31 \%$ by day 30 after neurectomy $(P<0 \cdot 05)$. By contrast, bone volume reduction induced by neurectomy in $\mathrm{kl} / \mathrm{kl}$ mice was approximately $9 \%$ by day 13 and $8 \%$ by day 30, indicating clearly the resistance of these animals to neurectomy-induced bone volume reduction (Fig. 2).

We next examined the effect of unloading due to neurectomy on the cortical bones in $\mathrm{kl} / \mathrm{kl}$ and $+/ \mathrm{kl}$ mice as their response to neurectomy may be different from that in trabecular bones. The morphology of cortex of the midshaft of femur in the plane perpendicular to the long axis of bone was investigated by two-dimensional $\mu \mathrm{CT}$ analysis. Thirty days after neurectomy, cortical bones in $+/ k l$ mice become thinner (Fig. 3) as described previously for wild-type mice. Quantification of these cross-sections of the cortical bones indicated that B.Ar in the neurectomized side femora (right) of $+/ \mathrm{kl}$ mice was reduced by $16 \%$ compared with that in the sham-operated side (left) (Fig. 4; $P<0 \cdot 05$ ). By contrast, no such reduction was observed in the cortical bone area of the femora of $k l / k l$ mice (Fig. 4, kl/kl). The values for bone area in loaded (left) side femora were similar between $\mathrm{kl} / \mathrm{kl}$ and $+/ \mathrm{kl}$ mice. No statistically significant difference was observed in the Ma.Ar, regardless of neurectomy and genotypes. Because the geometric shape and the size of the bones and the effect of the genotype might be affected by weight of the animals, we compared the body weight of the mice. The body weights (mean \pm standard deviation) of the $+/ k l$ mice and $\mathrm{kl} / \mathrm{kl}$ animals at the time of neurectomy were $18.7 \pm 3.6$ and $17 \cdot 7 \pm 1.5(\mathrm{~g})$ wherease those at the end of the 30-day period were $23 \cdot 1 \pm 2 \cdot 4$ and $22 \cdot 0 \pm 2 \cdot 6(\mathrm{~g})$ respectively. Thus, body weight is not a factor affecting the effects of neurectomy on the bones of the klotho mutant mice compared with the bones of $+/ k l$ mice. 


\section{$k l / k l$}

Sham-operated Neurectomized

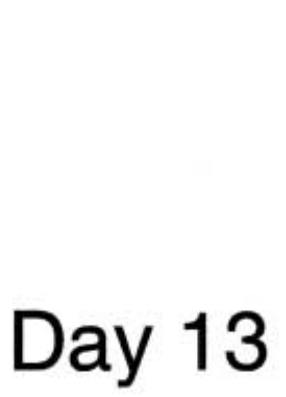

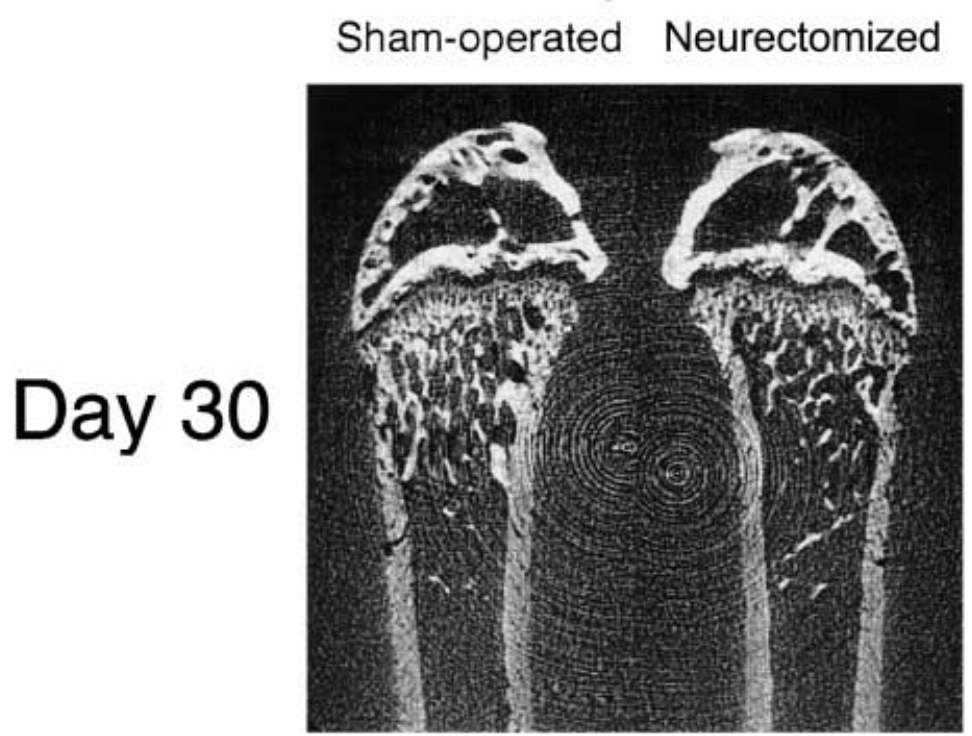

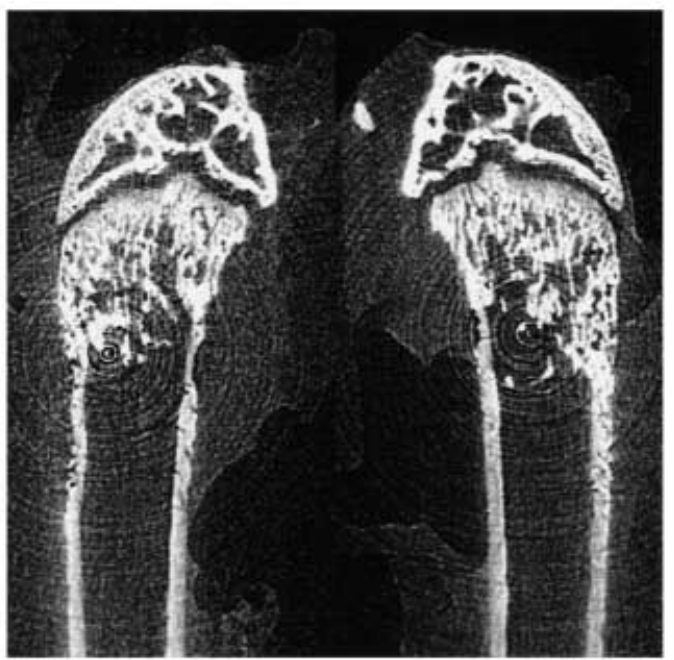

\section{$+/ k l$}

\section{Sham-operated Neurectomized}

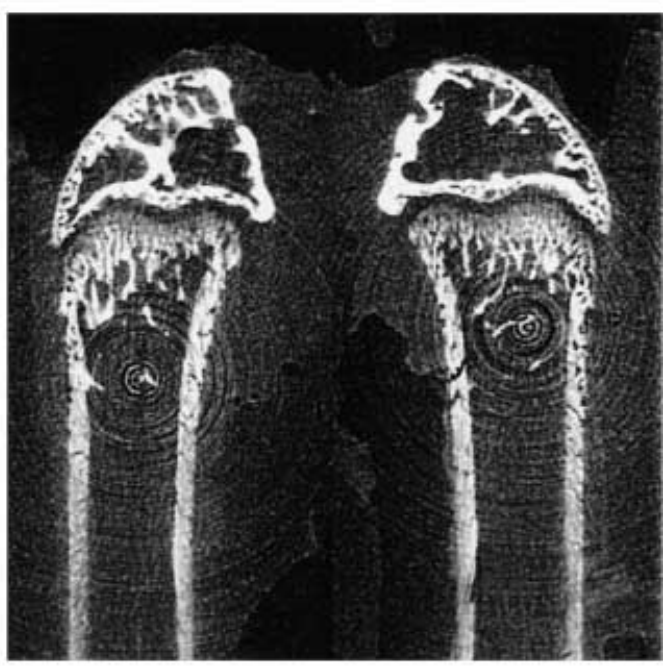

$+/ k l$

\section{Sham-operated Neurectomized}

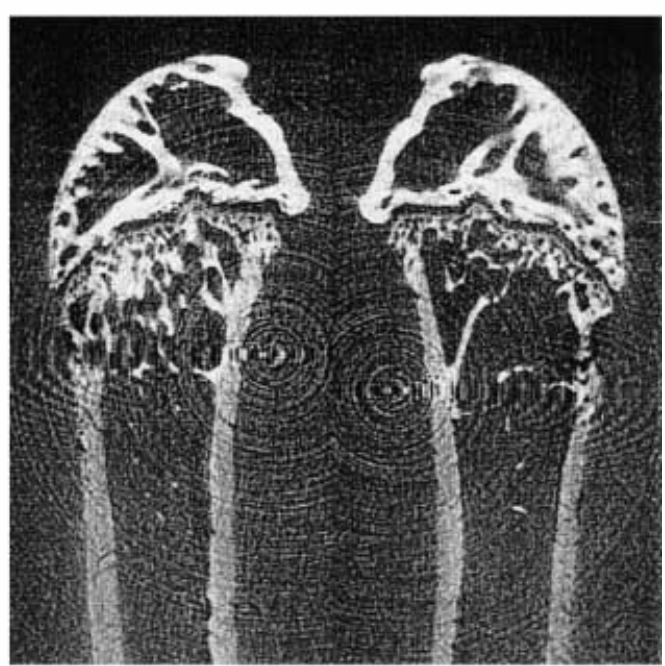

Figure 1 Two-dimensional $\mu \mathrm{CT}$ images of the sagittal plane of femora on day 13 and 30 after neurectomy. The trabecular bone pattern in the $+/ \mathrm{kl}$ neurectomized side femora became sparse compared to that in the sham-operated side. In $\mathrm{kl} / \mathrm{kl}$ mice there was no significant difference in the trabecular bone area at 30 days after neurectomy.

\section{Discussion}

In this paper we report that klotho mutant $(k l / k l)$ mice are resistant against bone volume reduction induced by unloading due to neurectomy. The resistance to bone volume reduction was clearly observed in the trabecular bone as well as in the cortical bone in the mid-shaft of the femur. Because unloading is one of the major causes of 


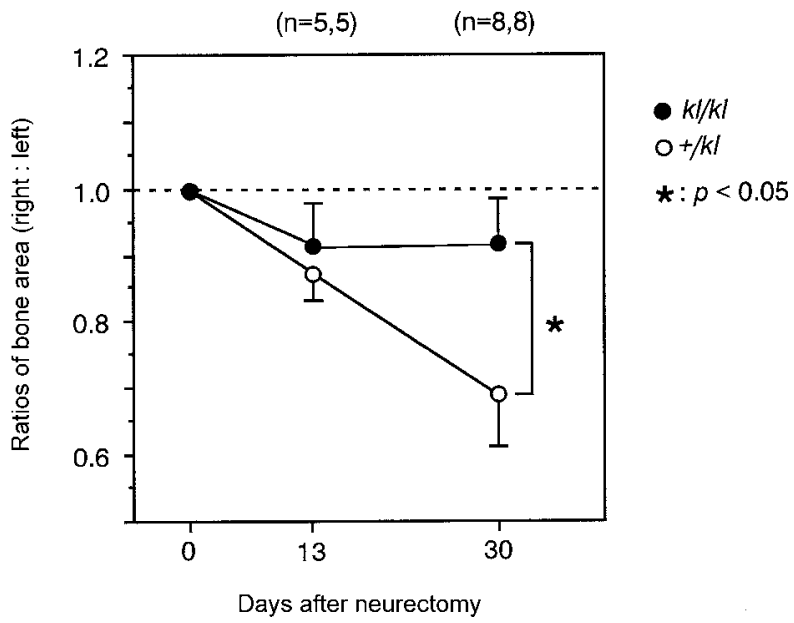

Figure 2 Ratios of trabecular bone volume (BV/TV) in the metaphyseal region of the right femora (neurectomized) over that of the left femora (sham-operated). Trabecular bone volume in $+/ \mathrm{kl}$ but not $\mathrm{kl} / \mathrm{kl}$ femora was significantly reduced at 30 days post-neurectomy. Values indicate mean \pm S.E.M. Asterisk $\left(^{*}\right)$ indicates that the difference is statistically significant $(P<0 \cdot 05) \cdot \mathrm{kl} / \mathrm{kl}=5,+/ \mathrm{kl}=5$ on day $13 ; \mathrm{kl} / \mathrm{kl}=8,+/ \mathrm{kl}=8$ on day 30 after operation.
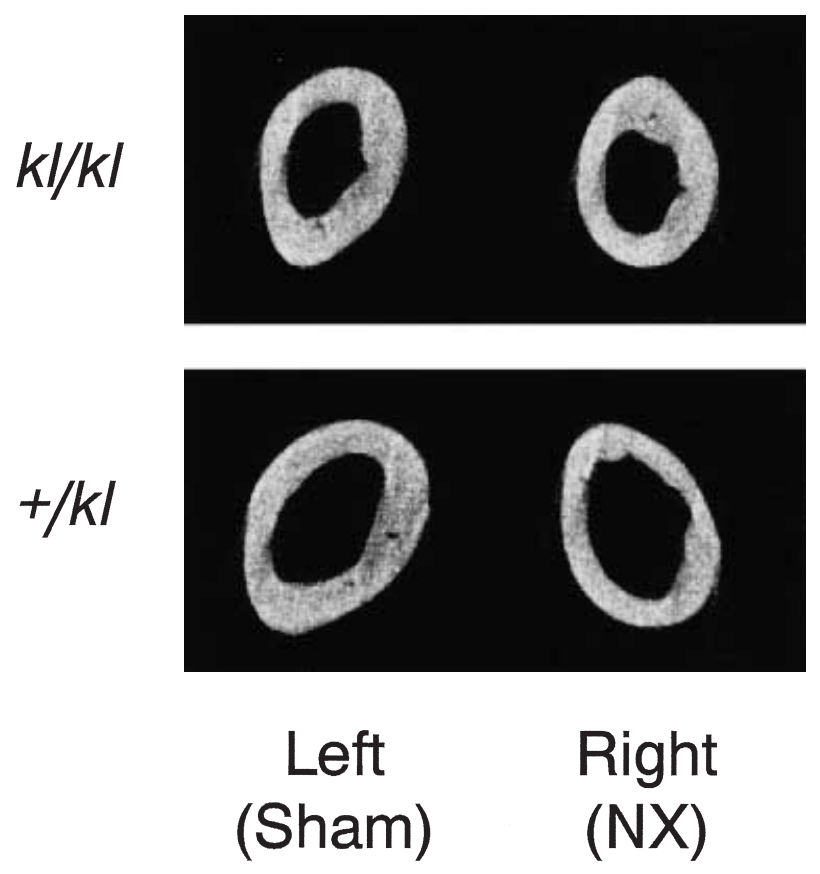

Figure 3 Two-dimensional tomographs of cross-section at mid-shaft of femora at 30 days post-neurectomy. The values for cortical bone area of $+/ k l$ femora were reduced by neurectomy, while those in $\mathrm{kl} / \mathrm{kl}$ mice were not altered by neurectomy. $\mathrm{kl} / \mathrm{kl}$ : klotho mutant mice, +/kl: heterozygous mutant mice, Right (NX): neurectomized, Left (Sham): sham-operated.

osteoporosis in bed-ridden patients or astronauts, it is important to understand the mechanism of the effects of unloading on bones to contemplate measures to treat or to

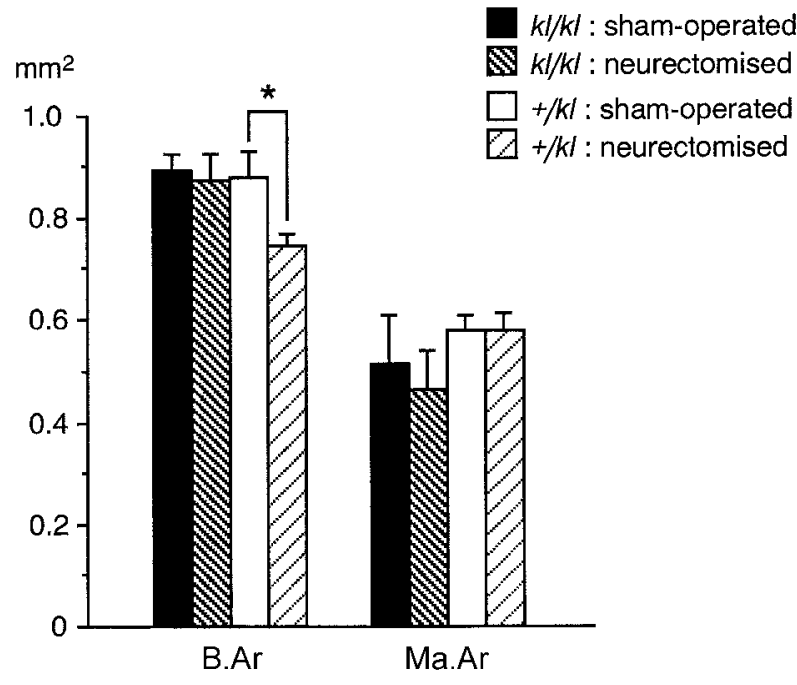

Figure 4 The klotho mutant mice were resistant to reduction in the cortical bone area of femora 30 days after neurectomy. Cortical bone area (B.Ar) of femora decreased significantly in $+/ k l$ mice, while $\mathrm{kl} / \mathrm{kl}$ femora were resistant to the loss of cortical bone. Asterisk $\left(^{*}\right)$ shows significant $(P<0 \cdot 05)$ differences. B.Ar: cortical bone area; Ma.Ar: bone marrow area.

prevent the diseases. Neurectomy-induced unloading is a model relevant to the bone loss in patients with paralysisinduced unloading. Our current data indicate that loss of the klotho gene product modulates the osteoporosis observed in the neurectomy model.

As neurectomy-induced bone volume reduction was prevented in klotho mutant mice, the loss of the klotho gene could either prevent increase in osteoclastic acitivity and/or could suppress the impairment in osteooblastic activity due to unloading induced by neurectomy. We previously observed that after bone marrow ablation $\mathrm{kl} / \mathrm{kl}$ mice can form new trabecular bone similarly to wild-type and $+/ k l$ mice, whereas bone resorption that occurs subsequent to bone formation in wild-type and $+/ k l$ mice is severely impaired in $\mathrm{kl} / \mathrm{kl}$ mice (Yamashita et al. 2000a). In the marrow ablation model, we observed that expression of receptor activator of nuclear factor kappa $\beta$ (RANK)L and RANK were normal but osteoprotegerin/ osteoclastogenesis inhibitory factor (OPG/OCIF) levels were significantly higher in $\mathrm{kl} / \mathrm{kl}$ mice than wild-type or $+/ k l$ mice. The resistant to bone resorption induced by unloading due to neurectomy could also be due, at least in part, to the relatively high levels of OPG/OCIF in the bone marrow tissues in $\mathrm{kl} / \mathrm{kl}$ mice. Marrow ablation data clearly indicated the presence of the impairment in bone resorption activity in klotho mutant mice; thus at least some of our observations could be due to the impairment in bone resorption. Whether the absence of the klotho gene could also suppress reduction in bone formation due to neurectomy is still to be determined.

It is not fully understood whether loading and unloading regulate calcium behavior via the alternation of the levels 
of systemic factors. Our data indicate that the klotho gene product could act in favor of unloading-induced bone loss and suggest that OPG/OCIF administration may prevent bone loss due to unloading at least in part. Because the klotho gene is expressed in extraskeletal tissues such as kidney, but not in bone, the klotho gene product could function as a humoral factor. In klotho mutant mice, a part of the gene product does not have a transmembrane domain and could therefore be released as a soluble form (Matsumura et al. 1998, Shiraki-Iida et al. 1998). Parabiosis experiments have supported this idea (Saito et al. 1998).

Although $+/ k l$ heterozygote mice could reveal phenotypes in some cases such as vascular response to certain stimuli (Saito et al. 1998), bone loss due to unloading by neurectomy was similar in wild-type and $+/ k l$ mice. In addition, osteopetrotic changes were not observed in heterozygote $(+/ k l)$ mice. Therefore, resistance to bone loss due to unloading requires homozygous mutation in the klotho gene locus.

It is known that tail suspension model is more preferable as an unloading model than neurectomy. However, we could not use this model as $\mathrm{kl} / \mathrm{kl}$ mice are susceptible to stress and can die when subjected to a major stress such as tail suspension. Therefore, the neurectomy model, which is less stressful, was chosen. As neurectomy could also induce many other stresses in addition to unloading, we cannot attribute the phenotype to the unloading alone. Nevertheless, $+/ k l$ heterozygote mice are still susceptible to bone loss by unloading similarly to wild-type mice; therefore, we assume that our observations on the reduction of bone loss in $\mathrm{kl} / \mathrm{kl}$ mice are at least in part due to the alteration in the bone metabolism in the $\mathrm{kl} / \mathrm{kl}$ mice.

In conclusion, mechanical unloading-induced bone loss requires the presence of the klotho gene products. Thus, the klotho gene product is acting as a factor favoring unloading-induced bone volume reduction.

\section{Acknowledgements}

This research was supported by a grant-in-aid received from the Ministry of Education (12557123, 12026212, 12215039, 0930734), TBRF, a grant from CREST of Japan Science and Technology Corporation (JST), and a grant from the Research for the Future Program of the Japan Society for the Promotion of Science (JSJP) (96100205) and NASDA (Japan Space Forum).

\section{References}

Kawaguchi H, Manabe N, Miyamura C, Chikuda H, Nakamura K \& Kuro-o M 1999 Independent impairment of osteoblast and osteoclast differentiation in klotho mouse exhibiting low-turnover osteopenia. Journal of Clinical Investigation 104 229-237.

Kodama Y, Dimai HP, Wergedal J, Sheng M, Malpe R, Kutilek S, Beamer W, Donahue LR, Rosen C, Baylink DJ \& Farley J 1999
Cortical tibial bone volume in two strains of mice: effects of sciatic neurectomy and genetic regulation of bone response to mechanical loading. Bone 25 183-190.

Kuro-o M, Matsumura Y, Aizawa H, Kawaguchi H, Suga T, Utsugi T, Ohyama Y, Kurabayashi M, Kaname T, Kume E, Iwasaki H, Iida A, Shiraki-Iida T, Nishikawa S, Nagai R \& Nabeshima YI 1997 Mutation of the mouse klotho gene leads to a syndrome resembling ageing. Science $\mathbf{2 7 8} \mathbf{4 5 - 5 1 .}$

Matsumura Y, Aizawa H, Shiraki-Iida T, Nagai R, Kuro-o M \& Nabeshima Y 1998 Identification of the human klotho gene and its two transcripts encoding membrane and secreted klotho protein. Biochemical and Biophysical Research Communications 242 626-630.

Morey ER \& Baylink DJ 1978 Inhibition of bone formation during space flight. Science 201 1138-1141.

Murakami H, Nakamura T, Tsurukami H, Abe M, Barbier A \& Suzuki K 1994 Effects of tiludronate on bone mass, structure, and turnover at the epiphyseal, primary, and secondary spongiosa in the proximal tibia of growing rats after sciatic neurectomy. Journal of Bone and Mineral Research 9 1355-1364.

Saito Y, Yamagishi T, Nakamura T, Ohyama Y, Aizawa H, Suga T, Matsumura Y, Masuda H, Kurabayashi M, Kuro-o M, Nabeshima Y \& Nagai R 1998 Klotho protein protects against endothelial dysfunction. Biochemical and Biophysical Research Communications 248 324-329.

Sakai A, Nakamura T, Tsurukami H, Okazaki R, Nishida S, Tanaka Y, Norimura T \& Suzuki K 1996 Bone marrow capacity for bone cells and trabecular bone turnover in immobilized tibia after sciatic neurectomy in mice. Bone 18 479-486.

Shen V, Liang XG, Birchman R, Wu DD, Healy D, Lindsay R \& Dempster DW 1997 Short-term immobilization-induced cancellous bone loss is limited to regions undergoing high turnover and/or modeling in mature rats. Bone 21 71-78.

Shiraki-Iida T, Aizawa H, Matsumura Y, Sekine S, Iida A, Anazawa H, Nagai R, Kuro-o M \& Nabeshima Y 1998 Structure of the mouse klotho gene and its two transcripts encoding membrane and secreted protein. FEBS Letters 424 6-10.

Simske SJ, Guerra KM, Greenberg AR \& Luttges MW 1992 The physical and mechanical effects of suspension-induced osteopenia on mouse long bones. Journal of Biomechanics 25 489-499.

Turner RT \& Bell NH 1986 The effects of immobilization on bone histomorphometry in rats. Journal of Bone and Mineral Research $\mathbf{1}$ 399-407.

Wakley GK, Baum BL, Hannon KS \& Turner RT 1988 The effects of tamoxifen on the osteopenia induced by sciatic neurotomy in the rat: a histomorphometric study. Calcified Tissue International 43 383-388.

Weinreb M, Rodan GA \& Thompson DD 1989 Osteopenia in the immobilized rat hind limb is associated with increased bone resorption and decreased bone formation. Bone 10 187-194.

Wronski TJ \& Morey ER 1982 Skeletal abnormalities in rats induced by simulated weightlessness. Metabolic Bone Disease and Related Research 4 69-75.

Yamashita T, Nifuji A, Furuya K, Nabeshima Y \& Noda M 1998 Elongation of the epiphyseal trabecular bone in transgenic mice carrying a klotho gene locus mutation that leads to a syndrome resembling aging. Journal of Endocrinology 159 1-8.

Yamashita T, Nabeshima Y \& Noda M 2000a High-resolution $\mu$ CT (micro-computed tomography) analyses of the abnormal trabecular bone structures in klotho gene mutant mice. Journal of Endocrinology $164239-245$.

Yamashita T, Yoshitake H, Tsuji K, Kawaguchi N, Nabeshima Y \& Noda M $2000 b$ Retardation in bone resorption after bone marrow ablation in klotho mutant mice. Endocrinology 141 438-445.

Zeng QQ, Jee WS, Bigornia AE, King J, Jr., D'Souza SM, Li XJ, Ma YF \& Wechter WJ 1996 Time responses of cancellous and cortical bones to sciatic neurectomy in growing female rats. Bone 19 13-21.

Received 7 August 2000

Accepted 24 October 2000 